\title{
A Note on Contracting Claw-Free Graphs
}

\author{
Jiří Fiala $\|^{1} \quad$ Marcin Kamiński非 Daniël Paulusma” \\ ${ }^{1}$ Department of Applied Mathematics, Charles University, Prague, Czech Republic \\ ${ }^{2}$ Instytut Informatyki, Uniwersytet Warszawski, Poland \\ ${ }^{3}$ School of Engineering and Computing Sciences, Durham University, United Kingdom \\ received $5^{\text {th }}$ Apr. 2012, revised $3^{\text {rd }}$ Apr. 2013, accepted 14 th Aug. 2013.
}

\begin{abstract}
A graph containment problem is that of deciding whether one graph called the host graph can be modified into some other graph called the target graph by using a number of specified graph operations. We consider edge deletions, edge contractions, vertex deletions and vertex dissolutions as possible graph operations permitted. By allowing any combination of these four operations we capture the following problems: testing on (induced) minors, (induced) topological minors, (induced) subgraphs, (induced) spanning subgraphs, dissolutions and contractions. We show that these problems stay NP-complete even when the host and target belong to the class of line graphs, which form a subclass of the class of claw-free graphs, i.e., graphs with no induced 4-vertex star. A natural question is to study the computational complexity of these problems if the target graph is assumed to be fixed. We show that these problems may become computationally easier when the host graphs are restricted to be claw-free. In particular we show that the $H$-CONTRACTIBILITY problem, which asks whether a given host graph contains a fixed target graph $H$ as a contraction, is polynomial-time solvable on claw-free graphs when $H$ is the 4-vertex path $P_{4}$, whereas on general graphs $P_{4}$-CONTRACTIBILITY is known to be NP-complete.
\end{abstract}

Keywords: claw-free graph, contractibility, containment relation

\section{Introduction}

Whether or not a graph $G$ contains a graph $H$ depends on the notion of containment we use; in the literature several natural definitions have been studied. Before we give a survey of existing work and present our own results, we first state some basic terminology.

\subsection{Terminology}

We consider undirected graphs with no loops and no multiple edges. We denote the vertex set and edge set of a graph $G$ by $V_{G}$ and $E_{G}$, respectively. If no confusion is possible, we may omit subscripts. We refer the reader to Diestel [5] for any undefined graph terminology.

\footnotetext{
†Email: fiala@kam.mff.cuni.cz. Supported by MŠMT ČR (LH12095), GAČR (P202/12/G061) and Royal Society (JP090172)

‡Email: mjk@mimuw. edu.pl.

§Email: daniel.paulusma@durham.ac.uk. Supported by EPSRC (EP/G043434/1) and Royal Society (JP090172).

1365-8050 @ 2013 Discrete Mathematics and Theoretical Computer Science (DMTCS), Nancy, France
} 


\begin{tabular}{|l|l|l|l|l|l|}
\hline Containment Relation & VD & ED & EC & VDi & Decision Problem \\
\hline minor & yes & yes & yes & yes & MINOR \\
\hline induced minor & yes & no & yes & yes & INDUCED MINOR \\
\hline topological minor & yes & yes & no & yes & TOPOLOGICAL MINOR \\
\hline induced topological minor & yes & no & no & yes & INDUCED TOPOLOGICAL MINOR \\
\hline contraction & no & no & yes & yes & CONTRACTIBILITY \\
\hline dissolution & no & no & no & yes & DISSOLUTION \\
\hline subgraph & yes & yes & no & no & SUBGRAPH ISOMORPHISM \\
\hline induced subgraph & yes & no & no & no & INDUCED SUBGRAPH ISOMORPHISM \\
\hline spanning subgraph & no & yes & no & no & SPANNING SUBGRAPH ISOMORPHISM \\
\hline isomorphism & no & no & no & no & GRAPH ISOMORPHISM \\
\hline
\end{tabular}

Tab. 1: Known containment relations in terms of the graph operations [11].

Let $e=u v$ be an edge in a graph $G$. The edge contraction of $e$ removes $u$ and $v$ from $G$, and replaces them by a new vertex adjacent to precisely those vertices to which $u$ or $v$ were adjacent. In the case that one of the two vertices, say $u$, has exactly two neighbors that in addition are nonadjacent, then we call this operation the vertex dissolution of $u$.

Table 1 surveys the known graph containment relations that can be obtained by combining vertex deletions (VD), edge deletions (EC), edge contractions (EC) and vertex dissolutions (VDi). For example, a graph $H$ is an induced minor of a graph $G$ if $H$ can be obtained from $G$ by a sequence of graph operations that include vertex deletions, vertex dissolutions and edge contractions, but no edge deletions. The corresponding decision problem, in which $G$ and $H$ form the ordered input pair $(G, H)$, is called INDUCED MINOR. The other rows in Table 1 are to be interpreted similarly.

Remark 1. If edge contractions are allowed then vertex dissolutions are allowed as well, because a vertex dissolution is a special case of an edge contraction. This means that the total number of different graph operation combinations is 12 .

Remark 2. As can be seen from Table 1 all but two combinations correspond to known relations. The remaining two combinations "no yes yes yes", and "no yes no yes" are equivalent to minors and topological minors, respectively, if we allow an extra operation that removes isolated vertices.

\subsection{Existing Results}

The problems in Table 1 except for GRAPH IsOMORPHISM are all known to be NP-complete (cf. [11, 21]). It is therefore natural to fix the graph $H$ (the target graph) in an ordered input pair $(G, H)$ and consider only the graph $G$ (the host graph) to be part of the input. We indicate this by adding " $H$-" to the names of the decision problems. For any fixed $H$, the problems $H$-DisSOLUtion, $H$-SUbGrapH ISOMORPHISM, $H$-INDUCED SUbGRAPH ISOMORPHISM, $H$-SPANNING SUBgRAPH ISOMORPHISM, and $H$-GRAPH ISOMORPHISM can be solved in polynomial time by brute force. A celebrated result by Robertson and Seymour [22] states that the problems $H$-MINOR and $H$-TOPOLOGICAL MinOR can be solved in cubic time and polynomial time, respectively, for every fixed graph $H$. The latter result has recently been improved to cubic time by Grohe et al. [12]. The computational complexity classifications (with respect to the fixed target graph $H$ ) of the remaining three problems $H$-INDUCED MinOR, $H$ IndUCED TOPOlOgicAl MinOR and $H$-CONTRACTIBILITY are still open. Many partial results are known, in particular for special graph classes. Below we briefly survey these. 
Fellows et al. [6] showed that the $H$-INDUCED MiNOR problem is NP-complete for a specific graph $H$ on 68 vertices. This is still the smallest known NP-complete case for $H$-INDUCED MINOR. They also showed that for every fixed graph $H$, the $H$-INDUCED MINOR problem can be solved in polynomial time on planar graphs. Later this result was extended by van 't Hof et al. [15] who showed that for every fixed planar graph $H$, the $H$-INDUCED MINOR problem is polynomial-time solvable on any minor-closed graph class not containing all graphs. Belmonte et al. [1] showed that for every fixed graph $H$, the $H$-INDUCED MINOR problem is polynomial-time solvable for chordal graphs.

Lévêque et al. [18] gave polynomial-time solvable and NP-complete cases for the $H$-INDUCED TOPOLOGICAL MINOR problem; small cases such as $H=K_{4}$ (the complete graph on 4 vertices) are still open. On the other hand, Fiala et al. [7] showed that for every fixed $H$, the $H$-INDUCED TOPOLOGICAL MiNOR problem can be solved in polynomial time on claw-free graphs, i.e., graphs with no induced 4-vertex stars.

Brouwer and Veldman [4] gave both polynomial-time solvable and NP-complete cases for the $\mathrm{H}$ CONTRACTIBILITY problem. One of their results is that this problem is already NP-complete for a graph $H$ on 4 vertices, namely when $H$ is fixed to be the 4-vertex path or the 4-vertex cycle. This research was later extended by Levin, Paulusma and Woeginger [19, 20] and van 't Hof et al. [15]. Kamiński, Paulusma and Thilikos [17] showed that for every fixed $H$, the $H$-CONTRACTIBILITY problem can be solved in polynomial time on planar graphs. By extending previous results [2, 10], Belmonte et al. [1] showed that for every fixed graph $H$, the $H$-CONTRACTIBILITY problem is polynomial-time solvable for chordal graphs.

\subsection{Our Results}

We focus on claw-free graphs and its proper subclass of line graphs, which are well studied, both within structural and algorithmic graph theory; we refer to Faudree, Flandrin, and Ryjáček [8] for a survey.

In Section 3 we show that all considered decision problems are NP-complete even under the further restriction that $G$ and $H$ are line graphs and both part of the input except for GRAPH IsOMORPHISM, which stays GRAPH ISOMORPHISM-complete for such input pairs. As such we can concentrate on the case when $H$ is fixed. Then the only two remaining problems are the $H$-INDUCED MINOR and $H$-CONTRACTIBILITY problem; as mentioned earlier on, the other eight $H$-containment problems are polynomial-time solvable on claw-free graphs for any fixed $H$.

In Section 4 we consider the question whether forbidding induced claws in the input graph makes the problem $H$-CONTRACTIBILITY computationally easier. Our motivation stems from the result on $H$-INDUCED TOPOLOGICAL MINOR, which is polynomial-time solvable on claw-free graphs for every fixed graph $H$ [7] while being NP-complete on general graphs already when $H$ is the complete graph on 5 vertices [18]. We provide a partially affirmative answer to this question as follows. In Section 4.1. we show that $P_{4}$-CONTRACTIBILITY, where $P_{4}$ denotes the 4-vertex path, is polynomial-time solvable for claw-free graphs. Recall that for general graphs, $P_{4}$-CONTRACTIBILITY is NP-complete [4]. In fact, we show that $H$-CONTRACTIBILITY is polynomial-time solvable on claw-free graphs for any fixed graph $H$ that is a so-called pileous clique, which is a special type of split graph. However, claw-freeness does not make the $H$-CONTRACTIBILITY problem tractable for all target graphs $H$ : in Section 4.2 we show that $P_{7}$-CONTRACTIBILITY is NP-complete even for line graphs. As a direct consequence, the LONGEST PATH CONTRACTIBILITY problem, which is that of determining the longest path to which a given graph can be contracted, is NP-hard for line graphs. This problem was introduced by van 't Hof, Paulusma and Woeginger [16] who showed that it is polynomial-time solvable for $P_{5}$-free graphs and NP-hard for $P_{6}$-free graphs, whereas Heggernes et al. [14] observe that this problem is polynomial-time solvable for 
chordal graphs.

In Section 5 we state some open problems and also shortly discuss the $H$-INDUCED MinOR problem for claw-free graphs.

\section{Preliminaries}

Let $G=(V, E)$ be a graph. We write $G[U]$ to denote the subgraph of $G$ induced by $U \subseteq V$, i.e., the graph on vertex set $U$ and an edge between any two vertices if and only if there is an edge between them in $G$. We say that $U$ is a clique if there is an edge in $G$ between any two vertices of $U$, and $U$ is an independent set if there is no edge in $G$ between any two vertices of $U$. Two disjoint sets $U, U^{\prime} \subseteq V$ are called adjacent if there exist vertices $u \in U$ and $u^{\prime} \in U^{\prime}$ such that $u u^{\prime} \in E$. A vertex $v$ is a neighbor of $u$ if $u v \in E$. We let $N(u)$ denote the set of neighbors of $u$. The degree of a vertex $u$ is its number of neighbors. The length $|P|$ of a path $P$ is the number of edges of $P$. The distance from a vertex $u$ to a vertex $v$ in $G$ is the length of a shortest path from $u$ to $v$ in $G$. We let $C_{n}, K_{n}$, and $P_{n}$ denote the cycle, complete graph, and path on $n$ vertices, respectively.

A graph $G=(V, E)$ is called $k$-connected if $G[V \backslash U]$ is connected for every set $U \subseteq V$ of at most $k-1$ vertices. A graph $G$ that is not connected is called disconnected. A $k$-vertex cut is a subset $S \subseteq V$ of size $k$ such that $G[V \backslash S]$ is disconnected. The vertex in a 1-vertex cut of a graph $G$ is called a cut vertex.

A star is a graph formed by joining each vertex of an independent set to an extra vertex called the centre vertex. A double star is formed by joining each vertex of an independent set to one of the two end-vertices of an extra edge called the centre edge. A pileous clique is a graph with the property that its vertex set can be decomposed into a clique and an independent set of degree-1 vertices. Note that pileous cliques form a subclass of split graphs. In particular, stars and double stars (including the $P_{4}$ ) are special cases of pileous cliques. A graph is claw-free if it has no induced subgraph isomorphic to the claw, i.e., the 4-vertex star $K_{1,3}=\left(\left\{a_{1}, a_{2}, a_{3}, b\right\},\left\{a_{1} b, a_{2} b, a_{3} b\right\}\right)$

The line graph of a graph $G$ with edges $e_{1}, \ldots, e_{p}$ is the graph $L(G)$ with vertices $u_{1}, \ldots, u_{p}$ such that there is an edge between any two vertices $u_{i}$ and $u_{j}$ if and only if $e_{i}$ and $e_{j}$ share one end vertex in $H$. We call $G$ the preimage of $L(G)$. Note that every line graph is claw-free. We also observe that $L\left(K_{3}\right)=L\left(K_{1,3}\right)=K_{3}$. However, $K_{3}$ is well-known to be unique in this perspective (see e.g. Harary [13]).

Let $G$ and $H$ be two graphs. An $H$-witness structure $\mathcal{W}$ is a vertex partition of $G$ into $\left|V_{H}\right|$ (nonempty) sets $W(x)$ called $H$-witness bags, such that

(i) each $W(x)$ induces a connected subgraph of $G$;

(ii) for all $x, y \in V_{H}$ with $x \neq y$, bags $W(x)$ and $W(y)$ are adjacent in $G$ if and only if $x$ and $y$ are adjacent in $H$;

By contracting all bags to single vertices we find that $H$ is a contraction of $G$ if and only if $G$ has an $H$-witness structure. We note that $G$ may have more than one $H$-witness structure. We call a bag that corresponds to a vertex of degree 1 in $H$ a leaf bag.

\section{When Both Host and Target Graph are Part of the Input}

We show the following result, which justifies why we fix the target graph $H$ in the remainder of our paper. 
Proposition 1 All problems in Table 1 are NP-complete for ordered input pairs $(G, H)$ where $G$ and $H$ are line graphs, except for the GRAPH ISOMORPHISM problem, which is GRAPH ISOMORPHISMcomplete for such input pairs.

Proof: Let $G$ be an $n$-vertex line graph, and $H$ be the $n$-vertex cycle; note that $H$ is a line graph and that $L(H)$ is an $n$-vertex cycle as well.

We observe that the statements that $G$ contains $H$ as a minor, topological minor, subgraph, or spanning subgraph, respectively are equivalent. The last statement (spanning subgraph) is equivalent to asking whether $G$ is Hamiltonian, which is an NP-complete problem even for line graphs as shown by Bertossi [3]. This means that we get the desired result for Minor, TOPOlOGiCAl MinOR, SubGRAPH ISOMORPHISM and SPANNING SUBGRAPH ISOMORPHISM. We note that $G$ contains $H$ as a spanning subgraph if and only if $L(G)$ contains $L(H)$ as an induced subgraph. Hence, from the same reduction, we obtain the desired result for INDUCED SUBGRAPH ISOMORPHISM.

We now show that $L(G)$ contains $L(H)$ as an induced subgraph if and only if $L(G)$ contains $L(H)$ as an induced minor. The forward implication holds by definition. To prove the backward implication, suppose that $L(G)$ contains $L(H)$ as an induced minor. By definition, $L(G)$ contains an induced subgraph $F$ that contains $L(H)$ as a contraction. We consider an $L(H)$-witness structure of $F$. Recall that $L(H)$ is a cycle on $n$ edges. We fix one edge between each pair of adjacent witness bags. Then these $n$ edges can be connected to each other via paths inside the witness bags. Hence $F$, and consequently, $L(G)$ contains an induced cycle $C$ on at least $n$ vertices. Note that $C$ corresponds to a (not necessarily induced) cycle in $G$ with the same number of vertices. Because $G$ has exactly $n$ vertices, we find that $C$ has exactly $n$ vertices. Consequently, $L(G)$ contains an induced $n$-vertex cycle, namely $C$, that is isomorphic to $L(H)$. Hence INDUCED MINOR is NP-complete when both the host and target graph are line graphs.

By a similar argument we can show that $L(G)$ contains $L(H)$ as an induced subgraph if and only if $L(G)$ contains $L(H)$ as an induced topological minor. In this way we get the desired result for INDUCED TOPOLOGICAL MiNOR as well.

For the CONTRACTIBILITY problem we refer to Corollary 6 which states that the $P_{7}$-ConTRACTIBILITY problem is NP-complete for line graphs; note that $H=P_{7}$ is a line graph, because $P_{7}=L\left(P_{8}\right)$.

The two remaining problems are DisSOLUTION and GRAPH ISOMORPHISM. We first consider the Dissolution problem. Let $G$ and $H$ be two graphs. For each vertex $u$ in $G$ that has degree at least 3 we do as follows. Suppose that $u$ has $p$ neighbors. We replace $u$ by $p$ new vertices, each adjacent to each other and to exactly one neighbor of $u$. Afterwards we do the same for each vertex $x$ in $H$ that is of degree at least 3 . We call the new graphs $G^{\prime}$ and $H^{\prime}$, respectively. We claim that $G^{\prime}$ and $H^{\prime}$ are line graphs. This can be seen as follows. For every maximal clique in $G^{\prime}$ we take a star with edges corresponding to the vertices of the maximal clique; note that some edges may belong to two stars. The resulting graph has $G^{\prime}$ as its line graph. By the same argument, $H^{\prime}$ is also a line graph. Moreover, $G$ contains $H$ as a dissolution if and only if $G^{\prime}$ contains $H^{\prime}$ as a dissolution. Because Dissolution is NP-complete, we then find that this problem stays NP-complete even when both the host and target graph are line graphs.

We now consider the GRAPH ISOMORPHISM problem. Let $G$ and $H$ be two arbitrary graphs on at least four vertices. Then $G$ and $H$ are isomorphic if and only if $L(G)$ and $L(H)$ are isomorphic, where the backward implication is due to the aforementioned observation that every connected line graph except for the graph $K_{3}$ has a unique preimage (see e.g. Harary [13]). This shows that GRAPH IsOMORPHISM stays GRAPH ISOMORPHISM-complete when $G$ and $H$ are restricted to line graphs. Hence we have proven Proposition 1 . 


\section{Contractions}

\subsection{Polynomial-Time Solvability}

We start with two useful lemmas, the second of which can be found in Levin et al. [19] but follows directly from the polynomial-time result on graph minors by Robertson and Seymour [22].

Lemma 2 Let $H$ be a connected graph on at least three vertices. If a graph $G$ contains $H$ as a contraction, then $G$ has an $H$-witness structure, in which every leaf bag consists of exactly one vertex.

Proof: Let $G$ be a graph that contains a connected graph $H$ on at least three vertices as a contraction. Then $G$ has an $H$-witness structure $\mathcal{W}$. Let $x$ be a vertex of $H$ with exactly one neighbor $y$. Suppose that $|W(x)| \geq 2$. By definition, there exists a vertex $u \in W(x)$ that is adjacent to $W(y)$. Let $D_{1}, \ldots, D_{p}$ be the connected components of $G[W(x) \backslash\{u\}]$. Because $|W(x)| \geq 2$, we find that $p \geq 1$. Let $v$ be a vertex of $D_{1}$ that is not a cut vertex of $G\left[D_{1}\right]$. We move all vertices of $W(x) \backslash\{v\}$ to $W(y)$. This results in a new $H$-witness structure of $G$. Because $H$ is a connected graph on at least three vertices, we did not increase the size of any other leaf bag. Hence, by repeating this procedure we obtain our desired witness structure.

Lemma 3 (|22]) Let $G$ be a graph and let $Z_{1}, \ldots, Z_{p} \subseteq V_{G}$ be $p$ specified pairwise disjoint sets such that $\sum_{i=1}^{p}\left|Z_{i}\right| \leq k$ for some fixed integer $k$. The problem of deciding whether $G$ contains $K_{p}$ as a contraction with $K_{p}$-witness bags $W_{1}, \ldots W_{p}$ such that $Z_{i} \subseteq W_{i}$ for $i=1, \ldots, p$ can be solved in polynomial time.

We are ready to state the first result in this section; recall that a pileous clique is a graph whose vertices of degree at least 2 form a clique, such as the $P_{4}$, and that already $P_{4}$-CONTRACTIBILITY is NP-complete for general graphs [4].

Theorem 4 If $H$ is a fixed pileous clique, then $H$-CONTRACTIBILITY is solvable in polynomial time on claw-free graphs.

Proof: Let $H$ be a pileous clique. If $H$ has one or two vertices, the problem is trivial even for general graphs. Suppose that $H$ has at least three vertices. We split the vertices of $H$ into two classes: $V_{1}$ containing all vertices of degree 1 and $V_{2}$ containing the remaining vertices, which induce a clique in $H$.

Let $G$ be a graph. By Lemma 2 we deduce that $G$ has an $H$-witness structure in which every leaf bag is of cardinality 1, should $G$ contain $H$ as a contraction. Hence we can do as follows. For each vertex $x \in V_{1}$, we guess a vertex $u_{x} \in V_{G}$ to form the corresponding leaf bag. We first check if the set of guessed $u_{x}$-vertices is independent. If not, then we discard the set of $u_{x}$-vertices. Otherwise, we proceed as follows. We observe that all neighbors of a vertex $u_{x}$ must go to the same bag which corresponds to the unique neighbor $y$ of $x$. Hence, we may contract any edge between two neighbors of $u_{x}$. Because $G$ is claw-free, $G\left[N\left(u_{x}\right)\right]$ consists of at most two connected components. This means that after performing the aforementioned edge contractions $u_{x}$ has at most two neighbors left in the resulting graph $G^{\prime}$, and these neighbors must be placed in the bag $W(y)$ corresponding to $y$. We now put all vertices that must go to $W(y)$ in the set $Z(y)$. Note that such a set can contain more than two vertices in case $y$ has more than one neighbor in $V_{1}$. If $y$ has no neighbor in $V_{1}$, then we set $Z(y):=\emptyset$. It can happen that a vertex is in more than one set $Z(y)$. In that case we discard this set of $u_{x}$-vertices (and we must guess some other set of $u_{x}$-vertices). Suppose that this is not the case, i.e., no vertex is in more than one set $Z(y)$. We have that $\sum_{y \in V_{2}}|Z(y)| \leq 2\left|V_{1}\right|$. 
We remove all vertices $u_{x}$ and are left to check whether the resulting graph $G^{\prime \prime}$ obtained from $G^{\prime}$ contains $K_{\left|V_{2}\right|}$ as a contraction with witness bags $W(y)$ such that $Z(y) \subseteq W(y)$ for each $y \in V_{2}$. Because $\sum_{y \in V_{2}}|Z(y)| \leq 2\left|V_{1}\right|$ is fixed as $H$ is fixed, this can be done in polynomial time due to Lemma 3 Because the number of different guesses for the sets of $u_{x}$-vertices is at most $n^{\left|V_{1}\right|}$, which is polynomial as $H$ is fixed, the theorem follows.

\subsection{NP-Completeness}

We now show that $P_{k}$-ContractiBility is NP-complete for all $k \geq 7$ even on line graphs. In order to do this we first reformulate the problem. Let $F=L(G)$ be the line graph of a graph $G$. Because no disconnected graph contains $P_{k}$ as a contraction, we may assume without loss of generality that $G$ is connected. For the same reason we assume that $G$ has at least seven vertices. This means that $F$ is not isomorphic to $K_{3}$. Consequently, there exists no graph $G^{\prime}$ different from $G$ with $L\left(G^{\prime}\right)=F$.

Observe that $F$ contains $P_{k}$ as a contraction if and only if the edges of $G$ can be partitioned into $k$ nonempty classes $E_{1}, \ldots, E_{k}$, such that each class $E_{i}$ induces a connected subgraph in $G$ and moreover, every edge in $E_{i}$ (which we also call an $E_{i}$-edge) may only be adjacent to edges in $E_{1}$ or in $E_{2}$ if $i=1$, edges in $E_{i-1}, E_{i}$, or $E_{i+1}$ if $2 \leq i \leq k-1$ and edges in $E_{k-1}$ or $E_{k}$ if $i=k$. We call this problem the $k$-Edge Partition problem. Clearly, $P_{k}$-COnTractibility on line graphs and $k$-Edge Partition are polynomially equivalent.

Theorem 5 The $k$-EDGE PARTITION problem is NP-complete for $k=7$.

Proof: We will reduce from the HyPERgRAPH 2-Colorability (H2C) problem, which is known to be NP-compete (cf. [9]). An instance of this problem consists of a set system $\mathcal{S}=\left\{S_{1}, \ldots, S_{m}\right\}$ over a ground set $Q=\left\{q_{1}, \ldots, q_{n}\right\}$. We may assume that $S_{i} \neq \emptyset$ for all $1 \leq i \leq m$, and that $S_{1} \cup \cdots \cup S_{m}=Q$. The question is whether $Q$ can be 2-colored , i.e., can be partitioned into two subsets $Q^{b}$ and $Q^{r}$, such that no set in $\mathcal{S}$ is monochromatic, i.e., only contains elements of $Q^{b}$ or of $Q^{r}$.

For a given set system $\mathcal{S}$ we construct a graph $G$ as follows. First we form a clique on vertices $q_{1}, \ldots, q_{n}$, representing the set $Q$. In the next step we insert $2 m$ new isolated edges $v_{j} w_{j}$ and $v_{j}^{\prime} w_{j}^{\prime}$ for $j=1, \ldots, m$. We add edges between vertices $q_{i}$ and $v_{j}, v_{j}^{\prime}$ respectively, to build two copies of the incidence graph for $\mathcal{S}$. Namely we insert new edges $q_{i} v_{j}$ and $q_{i} v_{j}^{\prime}$ if and only if $q_{i} \in S_{j}$. The construction of $G$ is finished by adding two new isolated edges $x y$ and $x^{\prime} y^{\prime}$ and by making $x$ connected to all $w_{j}$ and analogously $x^{\prime}$ to all $w_{j}^{\prime}$, see Figure 1

We claim that $G$ is a yes-instance of 7-EDGE PARTITION if and only if $\mathcal{S}$ can be 2-colored.

First suppose that $\mathcal{S}$ can be 2-colored. Let $Q^{b}$ and $Q^{r}$ denote the subsets of $Q$ colored by blue and red, respectively. We partition the edges of $G$ as follows:

- $E_{1}=\{x y\}$

- $E_{7}=\left\{x^{\prime} y^{\prime}\right\}$

- $E_{2}=\left\{x w_{1}, \ldots, x w_{m}\right\}$

- $E_{6}=\left\{x^{\prime} w_{j}^{\prime}, \ldots, x^{\prime} w_{m}^{\prime}\right\}$

- $E_{3}=\left\{w_{1} v_{1}, \ldots, w_{m} v_{m}\right\} \cup\left\{v_{j} q_{i} \mid q_{i} \in S_{j} \cap Q^{r}\right\} \cup\left\{q_{i} q_{i^{\prime}} \mid q_{i}, q_{i^{\prime}} \in Q^{r}\right\}$ 


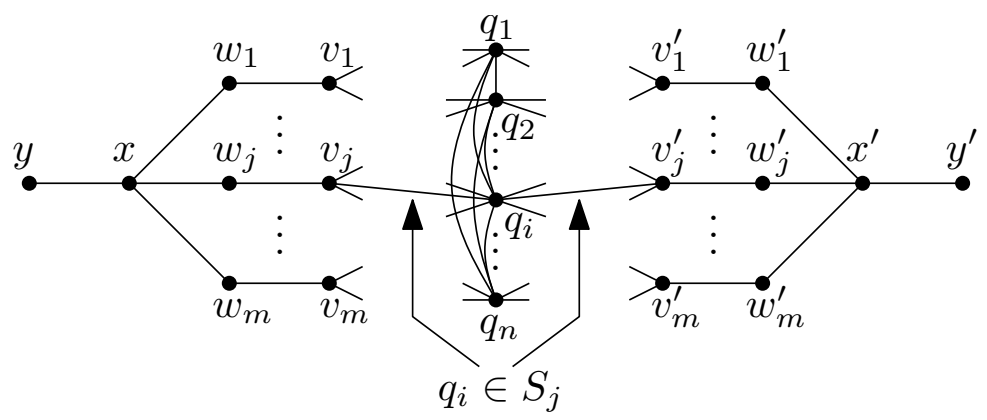

Fig. 1: The constructed graph $G$.

- $E_{5}=\left\{w_{1}^{\prime} v_{1}^{\prime}, \ldots, w_{m}^{\prime} v_{m}^{\prime}\right\} \cup\left\{v_{j}^{\prime} q_{i} \mid q_{i} \in S_{j} \cap Q^{b}\right\} \cup\left\{q_{i} q_{i^{\prime}} \mid q_{i}, q_{i^{\prime}} \in Q^{b}\right\}$

- $E_{4}=E_{G} \backslash\left(E_{1} \cup E_{2} \cup E_{3} \cup E_{5} \cup E_{6} \cup E_{7}\right)$

Obviously, $E_{1}, E_{2}, E_{6}$ and $E_{7}$ induce a connected subgraph. The $E_{3}$-edges also induce a connected subgraph, since every $E_{3}$-edge is incident with some (red) vertex $q_{i}$ and all such red vertices are connected in $E_{3}$. Analogously we get that $E_{5}$ induces a connected subgraph. Finally, the subgraph induced by $E_{4}$ contains a complete bipartite subgraph between red and blue $q$-vertices. Moreover, every $v_{j}$ is connected with an $E_{4}$-edge to some blue $q$-vertex, and every $v_{j}^{\prime}$ is connected with an $E_{4}$-edge to some red $q$-vertex. Hence $E_{4}$ induces a connected subgraph of $G$. Finally, the edge in $E_{1}$ is only adjacent to $E_{2}$-edges, and the edge in $E_{7}$ is only adjacent to $E_{6}$-edges, while the edges in all other partition classes are only adjacent to edges in their own partition class or to edges in the preceding and succeeding partition class. We conclude that $G$ is a yes-instance of 7-EDGE PARTITION.

Now suppose that $G$ is a yes-instance of 7-EDge PARTition. By construction of $G$, every $E_{1}$-edge must be incident with one of $\left\{x, x^{\prime}\right\}$ and every $E_{7}$-edge with the other vertex of $\left\{x, x^{\prime}\right\}$. Without loss of generality assume that $x$ belongs to every $E_{1}$-edge and that $x^{\prime}$ belongs to every $E_{7}$-edge. As $E_{3}$-edges cannot be incident to any $E_{1}$-edge, we get that $x$ is not incident with any $E_{3}$-edge. Analogously, $x^{\prime}$ is not incident with any $E_{5}$-edge. We also observe that an $E_{3}$-edge must be present in every path from $x$ to $x^{\prime}$ at distance at least 3 from $x^{\prime}$. Therefore, $E_{3}$-edges may only be incident with vertices $v_{j}$ and possibly $q_{i}$, but every $v_{j}$ is incident with at least one $E_{3}$-edge. As the $E_{3}$-edges induce a connected subgraph, we find in fact that every $v_{j}$ is connected to some $q_{i}$ by an $E_{3}$-edge. By symmetry of our construction, every $v_{j}^{\prime}$ is connected to some $q_{i}$ by an $E_{5}$-edge.

As $E_{3}$-edges and $E_{5}$-edges cannot be incident with the same vertex $q_{i}$, we may partition $Q$ into two parts, one part $Q^{r}$ containing those $q_{i}$ that are incident with an $E_{3}$-edge, and the other part $Q^{b}$ containing the remaining elements of $Q$. Because every $v_{j}$ is connected to some $q_{i}$ by an $E_{3}$-edge, every $S_{j}$ intersects $Q^{r}$. Analogously, as every $v_{j}^{\prime}$ is connected to some $q_{i}$ via an $E_{5}$-edge, every $S_{j}$ intersects $Q^{b}$ as well. For the sake of completeness note that there may be vertices $q_{i}$ incident only with $E_{4}$-edges, but we have chosen to put the corresponding elements $q_{i}$ to $Q^{b}$, although we could have distributed them arbitrarily. This completes the proof of Theorem 5

Corollary 6 The $P_{7}$-CONTRACTIBILITY problem is NP-complete for line graphs. 
Another example of a small graph $H$, for which $H$-ConTRACTIBILITY is NP-complete on claw-free graphs is $H=C_{6}$. This result can be shown by an analogous construction as the one used in the proof of Theorem 5 for the case $H=P_{7}$. The only difference is that the constructed graph will have the edge $x x^{\prime}$ instead of the edges $x y$ and $x^{\prime} y^{\prime}$. We omit a proof of this statement, since it would mimic the arguments of the proof of Theorem 5

\section{Future Work}

A computational complexity classification of the $H$-CONTRACTIBILITY problem for claw-free graphs is still open. For example, the cases $H=P_{5}$ or $H=P_{6}$ must still be resolved, as we cannot use the hardness reduction in Theorem 5 for them. We stress though that the aim of this note was to investigate whether claw-freeness of the input graph is useful for solving the $H$-CONTRACTIBILITY problem. We showed that $H$-CONTRACTIBILITY is NP-complete on claw-free graphs already for small graphs $H$, but also that there exist graphs $H$ for which $H$-CONTRACTIBILITY is polynomial-time solvable on claw-free graphs and NP-complete on general graphs. Hence, we conclude that claw-freeness helps but to a limited extent.

For the $H$-INDUCED MiNOR problem we can derive a similar result as Theorem 4 This result is not known for general graphs.

Proposition 7 The $H$-INDUCED MINOR problem is polynomial-time solvable on claw-free graphs whenever $H$ is a pileous clique.

Proof: Proposition 7 can be shown in a similar way as the corresponding result for $H$-CONTRACTIBILITY. We use the same algorithm as in the proof of Theorem 4 with the following modifications. First, we remove any common neighbors between two $u_{x}$-vertices if the corresponding $x$-vertices in $H$ have no common neighbor. Second, after removing the $u_{x}$-vertices, we apply Lemma 3 on the connected components of the remaining graph $G^{\prime \prime}$ instead of the whole $G^{\prime \prime}$. If we are successful with one such a component, then we are done. Otherwise we must guess a different set of $u_{x}$-vertices, as before.

Recall that the smallest known NP-complete case for $H$-INDUCED MINOR is a target graph $H$ on 68 vertices, as shown by Fellows et al. [6]. The gadget in their NP-completeness reduction is not clawfree, and the following problem is open. Does there exist a graph $H$ for which $H$-INDUCED MinOR is NP-complete for claw-free graphs?

We also recall that for any fixed $H$, the $H$-InduCED TopologiCAl Minor problem can be solved in polynomial time for claw-free graphs [7]. This means that $H$-INDUCED MINOR is polynomial-time solvable on claw-free graphs for any fixed $H$ that has maximum degree 2, because for such target graphs $H$ the two problems $H$-InduCED MinOR and $H$-INDUCED TOPOLOGICAL MinOr are polynomially equivalent. In particular this holds for $H=2 C_{3}$, where $2 C_{3}$ denotes the disjoint union of two 3-vertex cycles; determining the computational complexity of $2 C_{3}$-INDUCED MINOR for general graphs is a notoriously open problem.

\section{Acknowledgements}

We thank both anonymous reviewers for their useful comments that helped us to improve the readability of our paper. 


\section{References}

[1] R. Belmonte, P.A. Golovach, P. Heggernes, P. van 't Hof, M. Kaminski, and D. Paulusma. Finding contractions and induced minors in chordal graphs via disjoint paths. In: Proceedings of the 22nd International Symposium on Algorithms and Computation (ISAAC 2011), volume 7074 of Lecture Notes in Computer Science, pages 110-119, 2011.

[2] R. Belmonte, P. Heggernes, and P. van 't Hof. Edge contractions in subclasses of chordal graphs. Discrete Applied Mathematics, 160:999-1010, 2012.

[3] A.A. Bertossi. The edge hamiltonian path problem is NP-complete. Information Processing Letters, 13:157-159, 1981.

[4] A.E. Brouwer and H.J. Veldman. Contractibility and NP-completeness. Journal of Graph Theory, 11:71-79, 1987.

[5] R. Diestel. Graph Theory. Springer-Verlag, Electronic Edition, 2005.

[6] M.R. Fellows, J. Kratochvíl, M. Middendorf, and F. Pfeiffer. The Complexity of Induced Minors and Related Problems. Algorithmica, 13:266-282, 1995.

[7] J. Fiala, M. Kamiński, B. Lidický, and D. Paulusma. The $k$-in-a-path problem for claw-free graphs. Algorithmica, 62:499-519, 2012.

[8] R. Faudree, E. Flandrin, and Z. Ryjáček. Claw-free graphs-a survey. Discrete Mathematics, 164:87-147, 1997.

[9] M.R. Garey and D.S. Johnson. Computers and intractability. W.H. Freeman and Co., New York, 1979.

[10] P.A. Golovach, M. Kamiński, and D. Paulusma. Contracting a chordal graph to a split graph or a tree. In Proceedings of the 36th International Symposium on Mathematical Foundations of Computer Science (MFCS 2011), volume 6907 of Lecture Notes in Computer Science, pages 339-350, 2011.

[11] P.A. Golovach, M. Kaminski, D. Paulusma, and D.M. Thilikos. Containment relations in split graphs. Discrete Applied Mathematics, 160:155-163, 2012.

[12] M. Grohe, K. Kawarabayashi, D. Marx, and P. Wollan. Finding topological subgraphs is fixed-parameter tractable. In Proceedings of the 43rd ACM Symposium on Theory of Computing (STOC 2011), pages 479-488, 2011.

[13] F. Harary. Graph Theory. Addison-Wesley, Reading MA, 1969.

[14] P. Heggernes, P. van 't Hof, B. Lévěque, and C. Paul. Contracting chordal graphs and bipartite graphs to paths and trees. In: Proceedings of the 6th Latin-American Algorithms, Graphs and Optimization Symposium (LAGOS 2011), volume 37 of Electronic Notes in Discrete Mathematics, pages 87-92, 2011.

[15] P. van 't Hof, M. Kamiński, D. Paulusma, S. Szeider, and D.M. Thilikos. On graph contractions and induced minors. Discrete Applied Mathematics, 160:799-809, 2012.

[16] P. van 't Hof, D. Paulusma, and G.J. Woeginger. Partitioning graphs into connected parts. Theoretical Computer Science, 410:4834-4843, 2009.

[17] M. Kamiński, D. Paulusma, and D.M. Thilikos. Contractions of planar graphs in polynomial time. In Proceedings of the 18th Annual European Symposium on Algorithms (ESA 2010), volume 6346 of Lecture Notes in Computer Science, pages 122-133, 2010.

[18] B. Lévêque, D.Y. Lin, F. Maffray, and N. Trotignon. Detecting induced subgraphs. Discrete Applied Mathematics, 157:3540$3551,2009$.

[19] A. Levin, D. Paulusma, and G.J. Woeginger. The computational complexity of graph contractions I: polynomially solvable and NP-complete cases. Networks, 51:178-189, 2008.

[20] A. Levin, D. Paulusma, and G.J. Woeginger. The computational complexity of graph contractions II: two tough polynomially solvable cases. Networks, 52:32-56, 2008.

[21] J. Matoušek and R. Thomas. On the complexity of finding iso- and other morphisms for partial $k$-trees. Discrete Mathematics, 108:343-364, 1992.

[22] N. Robertson and P.D. Seymour. Graph minors. XIII. The disjoint paths problem. Journal of Combinatorial Theory, Series B, 63:65-110, 1995 